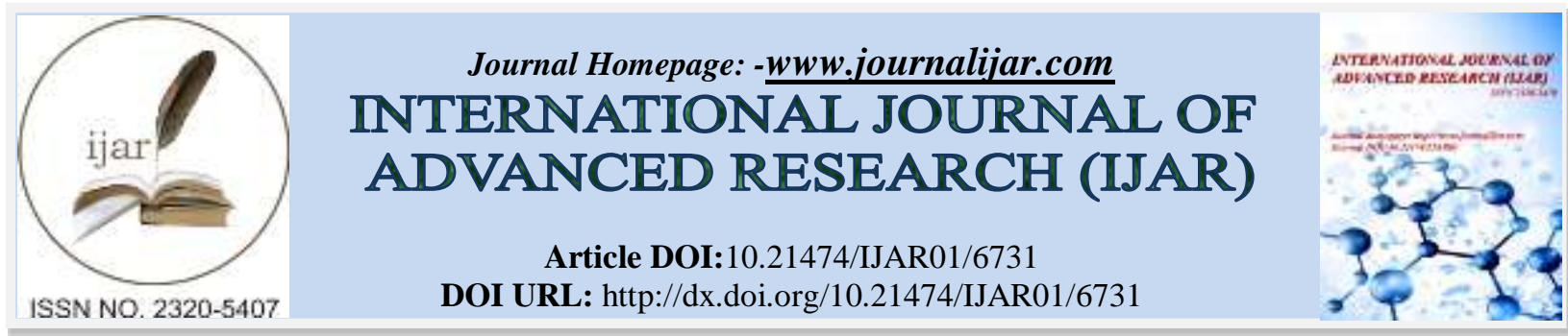

RESEARCH ARTICLE

\title{
TREATMENT OF TYPE 2 DIABETES MELLITUS WITH GLIMEPIRIDE AND METFORMIN BITHERAPY IN REAL CLINICAL PRACTICE IN THE GULF REGION: PATTERNS OF USE AND GLYCAEMIC CONTROL.
}

\author{
Reyaz Ahmed $^{1}$, Sameer Mohammed Al Shammari ${ }^{2}$, Leena Kapoor ${ }^{3}$ and Ahmed Mansour ${ }^{4}$ \\ 1. Internist-JCI accredited Hospital DXB, M.B.BS, and M.D. M.R.C.P. \\ 2. Head of the Endocrine Unit, Al Jahra Hospital, Kuwait. \\ 3. Prime Healthcare group, Prime medical centre, Dubai, UAE. \\ 4. Medical Manager Gulf Countries, Sanofi.
}

\section{Manuscript Info}

\section{Manuscript History}

Received: 12 January 2018

Final Accepted: 14 February 2018

Published: March 2018

Keywords:-

Diabetes, control, HbA1c, glimepiride, metformin.

\section{Abstract}

Patients with type 2 diabetes mellitus (T2DM) require regular monitoring and treatment adjustment to ensure sustained glycaemic control. The objective of this naturalistic study was to evaluate the effectiveness of bitherapy with glimepiride and metformin in T2DM patients inadequately controlled by monotherapy in everyday medical practice in the Gulf region, which has one of the highest prevalence rates of T2DM worldwide.

This was a prospective, observational study in four Gulf countries (Kuwait, Qatar, Oman and UAE). General practitioners enrolled adult patients with T2DM inadequately controlled (HB1Ac >7\%) on metformin or glimepiride monotherapy who were switched to bitherapy with glimepiride and metformin. Data were collected on HbA1c, weight, treatment and hypoglycaemia. The study duration was six months and the primary outcome variable was change in HbA1c (\%) over six months following initiation of bitherapy. 697 eligible patients were enrolled; 579 completed the six-month follow-up and were available for analysis and 292 patients $(50.4 \%)$ received bitherapy as a fixed-dose combination alone. During the study, mean $\mathrm{HbA} 1 \mathrm{c}$ fell from $8.92 \%$ to $7.08 \%$ (mean change: $1.84 \%$; $\mathrm{p}=0.03$ ). $46.1 \%$ of patients achieved their HbA1c target of $<7 \%$. No significant changes in weight were documented. The number of patients with symptomatic hypoglycaemia rose from $8(1.1 \%)$ in the three months before switching to bitherapy to $35(6.0 \%)$ in the three months following switch. Two patients discontinued glimepiride due to hypoglycaemia. At the last study visit, 57 patients $(9.8 \%)$ changed their dosing regimen and $35(6.0 \%)$ switched to another treatment, principally a gliptin.

Around half of patients who responded inadequately to monotherapy achieve glycaemic control targets within six months of switching to bitherapy with metformin and glimepiride. However, patients failing to achieve targets are infrequently moved to more intense treatment, suggesting that physician education on treat-to-target therapeutic strategies would be beneficial.

Copy Right, IJAR, 2018,. All rights reserved. 


\section{Introduction:-}

The Middle East has one of the highest prevalence rates for diabetes mellitus in the world, and has been rising continually over recent years due to lifestyle changes, notably sedentarisation. For example, Saudi Arabia and Kuwait have an age-adjusted prevalence of diabetes in adults of 20.0\%. Bahrain, Qatar, Egypt and the United Arab Emirates (UAE) also have prevalence rates $>15 \%$ (International Diabetes Foundation, 2015). For comparison, the regional prevalence of diabetes is $11.5 \%$ in North America and 7.3\% in Europe (International Diabetes Foundation, 2015). The large majority of these cases represent type 2 diabetes (T2DM) and the number of individuals in a prediabetic state of impaired glucose tolerance is also high (14\% in Saudi Arabia). Given the high prevalence of T2DM and the disease burden attributable to associated morbidity and mortality, management of diabetes in the Middle East represents a major public health issue.

If not treated adequately, patients with T2DM develop disabling and potentially life-threatening microvascular and macrovascular complications. However, use of antidiabetic medication to ensure adequate glycaemic control can markedly reduce the risk of developing these complications, as well as reducing mortality (UK Prospective Diabetes Study Group, 1998). The major challenge in the management of T2DM is that glycaemic control tends to escape over time, necessitating an intensification of treatment. For this reason, treatment algorithms have been developed to help physicians optimise glycaemic control over time in their patients. These algorithms recommend a 'treat-totarget' approach, in which glycosylated haemoglobin (HbA1c) is monitored regularly and a treatment changed to a more intense one if $\mathrm{HbAlc}$ rises above a pre-specified target. In particular, practice guidelines have been published by the American Diabetes Association and the European Association for the Study of Diabetes in 2009 (Nathan et al., 2009), subsequently updated in 2012 and 2015 (Inzucchi et al., 2012, Inzucchi et al., 2015). These guidelines recommend metformin as first-line monotherapy, unless contraindicated, in patients in whom glycaemic control, defined as $\mathrm{HbAlc} \leq 7 \%$, cannot be achieved by lifestyle modifications alone. It is recommended to measure HbAlc quarterly and to switch patients who do not achieve the target of $\mathrm{HbAlc} \leq 7 \%$ to bitherapy with another oral antidiabetic drug (OAD) or to insulin depending on the patient profile. In patients who still fail to achieve adequate control on bitherapy, a third OAD or insulin should be added. The justification for sequential add-on treatment is that the complementary pharmacological actions of different antidiabetic agents are expected to be synergistic in lowering blood glucose.

Sulphonylureas remain the class of OADs that are most frequently associated with metformin in bitherapy regimens. These drugs are well established, inexpensive and have a well-characterized safety profile. In patients who fail metformin therapy, clinical studies have demonstrated that between $44 \%$ and $66 \%$ patients will achieve glycaemic control following addition of sulphonylurea (Esposito et al., 2011). However, little information is available on the effectiveness of metformin and sulphonylurea bitherapy in real-world treatment settings.

We therefore decided to conduct a study in order to evaluate the effectiveness of combination treatment with glimepiride and metformin in T2DM patients inadequately controlled by oral monotherapy with one or the other agent in every day medical practice in the Gulf region. The primary objective of the study was to evaluate the change in HbAlc between baseline and following six months of combination therapy. Secondary outcomes included evaluation of the patterns of use of antidiabetic medication, the incidence of hypoglycaemia and weight change, treatment adherence and safety.

\section{Methods:- \\ Study design:-}

A regional non-interventional, prospective, multicentre, non-comparative observational study was conducted to collect information on the treatment of T2DM in real-life clinical practice in four countries in the Gulf region (Kuwait, Qatar, Oman and UAE). The study was conducted by a random sample of general practitioners [GPs] in each participating country. Given the naturalistic framework of the study, no fixed study visits were scheduled and visits were performed according to the routine clinical practice of the participating GP and the needs of the patient. The follow-up period for each individual patient was six months following initiation of combination treatment. The study was conducted between September 2012 and September 2014. 


\section{Participating physicians:-}

Participating physicians were selected by simple random sampling of a national listing of all GPs in each country and invited to participate by telephone. A target number of participating GPs was defined for each country based on the population size (fifteen from UAE, thirteen from Kuwait, five from Qatar and four from Oman). It was verified that contacted GPs who agreed to participate regularly treated patients with diabetes and had the necessary infrastructure to conduct the study.

\section{Study population:-}

Each participating centre was expected to enroll twenty consecutive patients. All potentially eligible subjects coming to the clinic were screened for inclusion in the study and those fulfilling the eligibility criteria were enrolled. Adult (>18 years) T2DM patients were eligible for inclusion if (i) they were inadequately controlled for at least three months on metformin or glimepiride monotherapy in the opinion of the treating GP, objectivized by an HB1 Ac >7\% and (ii) their treating GP had decided to prescribe them a combination of glimepiride and metformin. Pregnant and breastfeeding women, patients taking insulin, patients previously treated with another sulphonylurea, patients with hypersensitivity to the study medications and patients participating in another study were excluded.

\section{Data collection:-}

Data were collected at thee study visits at the inclusion site. These were the baseline visit at which combination therapy was initiated (V1), a follow-up visit at the end of three months \pm 20 days (V2) and an end-of-study visit at the end of six months \pm 20 days after combination treatment initiation (V3). Data were collected at each study visit on a paper case report form. All data collected was reviewed centrally and any inconsistencies or missing information were queried with the study site. Hypoglycaemic episodes were documented retrospectively at the study visits and classified as symptomatic and asymptomatic episodes. Symptomatic episodes were defined as events with clinical symptoms that are considered to result from hypoglycaemia, whether this was documented by a low plasma glucose level or not. Asymptomatic hypoglycaemia was defined as a measured plasma glucose level $\leq 70 \mathrm{mg} / \mathrm{dl}$ not associated with typical symptoms of hypoglycaemia. It should be noted that systematic self-monitoring of plasma glucose was not required by the study protocol, consistent with the naturalistic framework of the study. Severe symptomatic hypoglycaemia was defined as an event requiring the assistance of another person, associated with a measured plasma glucose $<36 \mathrm{mg} / \mathrm{dl}$ or for which neurological recovery occurred attributable to restoration of plasma glucose following carbohydrate administration. Serious hypoglycaemia was defined as a symptomatic hypoglycaemic event which led to loss of consciousness, a visit to an Emergency Department, hospitalisation or which otherwise fulfilled the definition of a serious adverse event.

\section{Outcome variables:-}

The primary outcome variable was the change in HbA1c (\%) between V1 and V3. The change between V1 and V2 and between V2 and V3 was also determined. The proportion of patients who achieved HbA1c <7\% was calculated. Weight was measured at each study visit and the change in weight between V1 and V2/V3 determined.

\section{Statistical analysis:-}

The number of patients to be included in the study was determined by a priori power calculations. The study hypothesis was that the reduction in HbAlc following the start of combination therapy would be $20 \%$. In order to detect such a change with an $\alpha$ risk of 0.05 and a $\beta$ risk of 0.05 , then it would be required to evaluate a sample of 651 patients. Assuming a drop-out rate of $20 \%$, then 782 patients would be required. The target sample size was thus set at 800 patients.

Three study populations were considered. The enrolled population, corresponding to all subjects included in the study, was considered for evaluation of safety. The eligible population corresponded to all included subjects with no violation of the eligibility criteria. The efficacy population, corresponding to all included eligible patients with an exploitable determination of HbA1c at V3.

The presentation of the results is principally descriptive. Continuous variables are presented as mean values \pm standard deviation (SD) or median values [range] as appropriate. Categorical variables are presented as frequency counts and percentages. For the primary outcome variable (change in HBA1c between V1 and V3), inferential statistical testing was performed using a paired Student's $t$-test. A two-tailed probability level of 0.05 was considered to be statistically significant. All statistical analyses were performed using SSPS version 21 software. 


\section{Ethics:-}

This study was performed in compliance with the Guidelines for Good Epidemiology Practice and with international and national laws and regulations regarding medical research. The study was submitted to, and approved by, the relevant institutional review boards of each participating country. All patients were informed about the objectives and procedures of the study and provided written informed before participating in the study.

\section{Results:-}

Participants:-

A total of 36 sites participated in the study and 722 patients were enrolled in the study. The majority of patients were recruited in Kuwait and UAE (Figure 1). Of the enrolment population, 25 patients were ineligible (3.4\%), principally since inadequate glycaemic control (HB1 Ac $>7 \%$ ) had not been demonstrated prior to or at baseline. The remaining 697 patients constituted the eligible population. Of these, 118 patients were excluded from the efficacy analysis (16.9\%), principally because the final study visit (V3) fell without the pre-specified time window (>140 to $<210$ days from baseline visit) or due to loss to follow-up. The remaining 579 patients constituted the efficacy population. The flow of patients through the study is presented in Figure 1.

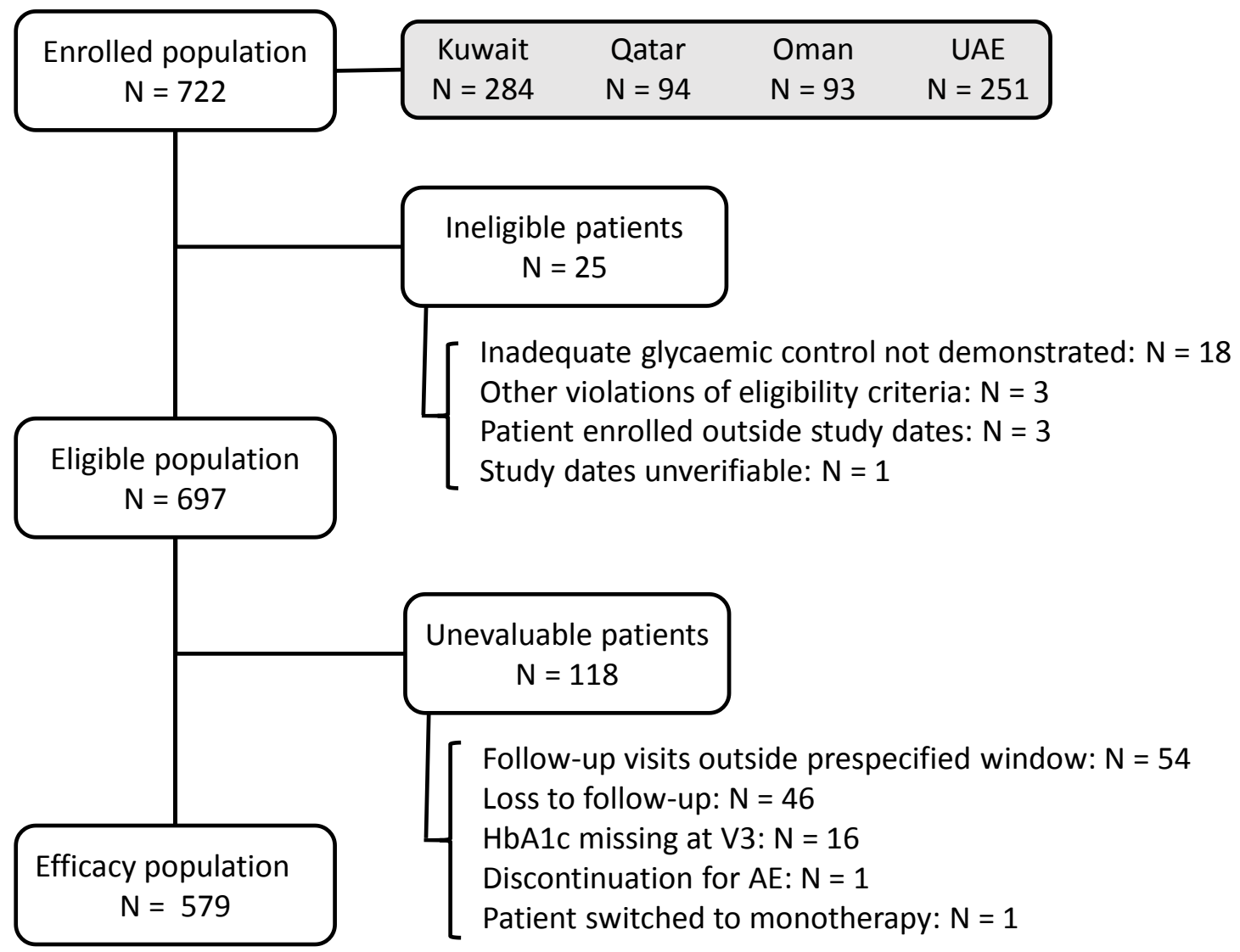

Figure 1:- Patient disposition.

AE: adverse event; HbA1c: glycosylated haemogolobin A1c; UAE: United Arab Emirates.

The demographic and clinical features of the eligible population are presented in Table 1. The mean age of the patients was 46 years in both men and women, with men accounting for three-quarters of the sample. At inclusion, patients were on average relatively recently diagnosed, with a mean time since diagnosis of 42 months and a mean duration of OAD therapy of 40 months. However, a wide range in values was observed for both these variables (up to 27 years). Metformin accounted for $85 \%$ of the previous monotherapy, with the dose of $2 \mathrm{mg}$ being the most commonly prescribed. Around $1 \%$ of the patients had presented episodes of symptomatic hypoglycaemia in the previous three months, although none of these episodes were considered severe or serious. The presence or absence 
of diabetic complications and comorbidities was investigated and documented on the case report form in $>95 \%$ of the eligible population. Peripheral neuropathy was the most frequently documented diabetic complication, followed by retinopathy and nephropathy. Hypertension and dyslipidaemia were documented in around one-third of the subjects.

\begin{tabular}{|c|c|}
\hline Age (years) & $N=697$ \\
\hline Mean \pm SD & $46 \pm 8$ \\
\hline Range & $27-75$ \\
\hline Gender & $N=696$ \\
\hline Men, n (\%) & $511(73.4 \%)$ \\
\hline Women, n (\%) & $185(26.6 \%)$ \\
\hline \multicolumn{2}{|l|}{ Time since diagnosis (months) } \\
\hline Mean \pm SD & $42 \pm 43$ \\
\hline Range & $3-334$ \\
\hline \multicolumn{2}{|l|}{ OAD treatment at baseline } \\
\hline Duration (mean $\pm \mathrm{SD})$ & $40 \pm 45$ \\
\hline Duration (range) & $3-328$ \\
\hline Metformin only & $589(84.5 \%)$ \\
\hline Glimepiride only & $98(14.1 \%)$ \\
\hline Metformin then switched to glimepiride or vice versa & $10(1.4 \%)$ \\
\hline Mean Metformin dose & $1500 \mathrm{mg}$ \\
\hline Mean glimepiride dose & $2 \mathrm{mg}$ \\
\hline Episodes of hyperglycaemia in previous 3 months & $N=687$ \\
\hline Symptomatic hypoglycaemia, n (\%) & $8(1.1 \%)$ \\
\hline Asymptomatic hypoglycaemia, n (\%) & $1(0.1 \%)$ \\
\hline Documented diabetic complications & (a) \\
\hline Peripheral neuropathy & $82(12.1 \%)$ \\
\hline Retinopathy & $37(5.5 \%)$ \\
\hline Nephropathy & $37(5.4 \%)$ \\
\hline Coronary artery disease & $20(3.0 \%)$ \\
\hline Other & $2(0.3 \%)$ \\
\hline Documented comorbidities & (a) \\
\hline Hypertension & $210(30.6 \%)$ \\
\hline Dyslipidaemia & $252(37.3 \%)$ \\
\hline
\end{tabular}

Table 1:- Patient characteristics (eligible population).

Percentages are reported with respect to the number of observed cases. OAD: oral antidiabetic drug. (a) The proportion of patients for whom diabetic complications and comorbidities were not documented ranged from these 11 for hypertension (1.6\% of patients) to 29 for coronary artery disease $(4.2 \%)$.

\section{Treatment:-}

Around half of patients received glimepiride and metformin as a fixed-dose combination and the other half as a free combination. The median dose of metformin was higher when free combinations were prescribed compared to fixed-dose combinations (Table 2). Twenty-two patients (3.7\%) were prescribed a fixed-dose combination with addon metformin.

\begin{tabular}{|c|l|}
\hline Free combination of glimepiride and metformin & $260(44.9 \%)$ \\
\hline Daily dose of glimepiride (mg; median[range]) & $2 \mathrm{mg}[1-6] \mathrm{mg}$ \\
\hline Dosing frequency of glimepiride (modal) & Once daily \\
\hline Daily dose of metformin (mg; median[range]) & $1500[500-3000]$ \\
\hline Dosing frequency of metformin (modal) & Twice daily \\
\hline Fixed dose combination of glimepiride and metformin (alone) & $292(50.4 \%)$ \\
\hline Daily dose of glimepiride/metformin (mg; median[range]) & $2 / 500[1 / 250-6 / 1500]$ \\
\hline Dosing frequency (modal) & Twice daily \\
\hline
\end{tabular}




\begin{tabular}{|l|l|}
\hline Fixed dose combination with add-on metformin & $22(3.8 \%)$ \\
\hline Daily dose of fixed combination (mg; median[range]) & $2 / 500[2 / 500-4 / 1000]$ \\
\hline Dosing frequency (modal) & Once daily \\
\hline Additional daily dose of metformin (mg; median[range]) & $1000[500-1500]$ \\
\hline $\begin{array}{l}\text { Mean daily dose of FDC in patients who were given additional metformin } \\
(\mathrm{n}=22)\end{array}$ & OD \\
\hline
\end{tabular}

Table 2:- Oral antidiabetic treatment at baseline (V1) in the efficacy population.

Data on treatment were missing for five patients.

At the second study visit three months after initiating bitherapy with glimepiride and metformin, the dosing regimen was changed in 115 patients prescribed free combinations (44.2\%) and 111 of those prescribed fixed-dose combinations (36.5\%). In the majority of cases, this involved an increase in dose of glimepiride or, for patients with free combinations, passage from once a day metformin to twice daily. In addition, 61 patients received an add-on oral therapy at Visit 2, principally DPP-4 inhibitors (52 patients), five patients were prescribed add-on insulin and three add-on GLP-1 analogues.

At the third study visit, 35 patients initially prescribed free combinations (13.5\%) and 22 prescribed fixed-dose combinations $(7.2 \%)$ changed their dosing regimen, principally to a higher dose. Thirty-one patients received an add-on therapy with another OAD (DPP-4 inhibitors in 23 cases) and four patients received add-on insulin.

\begin{tabular}{|l|l|l|l|}
\hline Group & Baseline & V2 & V3 \\
\hline $\begin{array}{l}\text { Free combination of Glimepiride and Metformin (Mean Daily } \\
\text { Dose) }\end{array}$ & $2 \mathrm{mg} / 1500 \mathrm{mg}$ & $\begin{array}{l}3 \text { mg/1700 } \\
\mathrm{mg}\end{array}$ & $\begin{array}{l}4 \\
\mathrm{mg} / 2000\end{array}$ \\
\hline $\begin{array}{l}\text { Fixed dose combination of Glimepiride + Metformin (Mean Daily } \\
\text { Dose) }\end{array}$ & $2 \mathrm{mg} / 1000 \mathrm{mg}$ & $2 \mathrm{mg} / 1000 \mathrm{mg}$ & $4 \mathrm{mg} / 1000 \mathrm{mg}$ \\
\hline
\end{tabular}

Table 3:- The mean daily dose at baseline and at study end in the 2 groups (free and Fixed Dose Combination)

\section{Compliance:-}

Compliance with antidiabetic treatment at the final study visit was rated by the investigator as very good in 421 patients (72.7\% of the efficacy population), good in 128 patients $(22.1 \%)$, sufficient in 21 patients (3.6\%) and insufficient in nine patients (1.6\%).

\begin{tabular}{|l|l|l|l|}
\hline \multicolumn{2}{|l|}{ Overall compliance of prescribed OAD as judged by the Investigator } & Free combination & FDC \\
\hline Very Good, $\mathrm{n}(\%)$ & $422(72.6 \%)$ & $194(33.4 \%)$ & $228(39.2 \%)$ \\
\hline Good, $\mathrm{n}(\%)$ & $129(22.2 \%)$ & $52(8.9 \%)$ & $77(13.3 \%)$ \\
\hline Sufficient, $\mathrm{n}(\%)$ & $21(3.6 \%)$ & $15(2.6 \%)$ & $6(1.0 \%)$ \\
\hline Not sufficient, $\mathrm{n}(\%)$ & $9(1.5 \%)$ & $7(1.2 \%)$ & $2(0.3 \%)$ \\
\hline
\end{tabular}

Table 4:- Compliance of prescribed OAD as judged by the Investigator in the 2 groups

\section{Glycaemic control:-}

Over the course of the study mean HbAlc levels fell from $8.92 \%$ to $7.08 \%$, a mean change of $1.84 \%(p=0.03)$. A significant decrease in HbAlc was already observable at the second study visit (Table ). At the end of the study, $46.1 \%$ of the previously uncontrolled patients had achieved their HbA1c target of $<7 \%$. 


\begin{tabular}{|c|l|l|l|}
\hline & $\begin{array}{l}\text { Baseline } \\
(\mathrm{V} 1)\end{array}$ & $\begin{array}{l}\text { Three months visit } \\
(\mathrm{V} 2)\end{array}$ & Six month visit (V3) \\
\hline HbA1c (\%; mean \pm SD) & $8.92 \pm 1.12$ & $7.78 \pm 0.78$ & $7.08 \pm 0.63$ \\
\hline Change from baseline (\%) & - & $\begin{array}{l}1.14 \\
(p=0.047)\end{array}$ & $\begin{array}{l}1.84 \\
(p=0.003)\end{array}$ \\
\hline HbA1c <7\% (n,\%) & None & $53(9.1 \%)$ & $267(46.1 \%)$ \\
\hline Weight $(\mathrm{kg}$; mean \pm SD) & $81.7 \pm 12.2$ & $81.3 \pm 11.8$ & $81.0 \pm 11.6$ \\
\hline Change from baseline (kg) & - & $\begin{array}{l}0.48 \\
(p=0.64)\end{array}$ & $\begin{array}{l}0.71 \\
(p=0.71)\end{array}$ \\
\hline
\end{tabular}

Table 5:-HbA1c and weight over the course of the study.

\section{Weight change:-}

Changes in body weight over the course of the study were minimal (Table 5).

\section{Hypoglycaemia:-}

During the course of the study, symptomatic hypoglycaemic episodes were reported by 35 patients during the first three months of bitherapy and by 45 patients during the second three month period. The corresponding figures for asymptomatic episodes were 7 and 18 respectively. No serious or severe episodes were documented. The mean number of episodes was five in the first three-month period and four in the second. However, some patients experienced more than one episode per week.

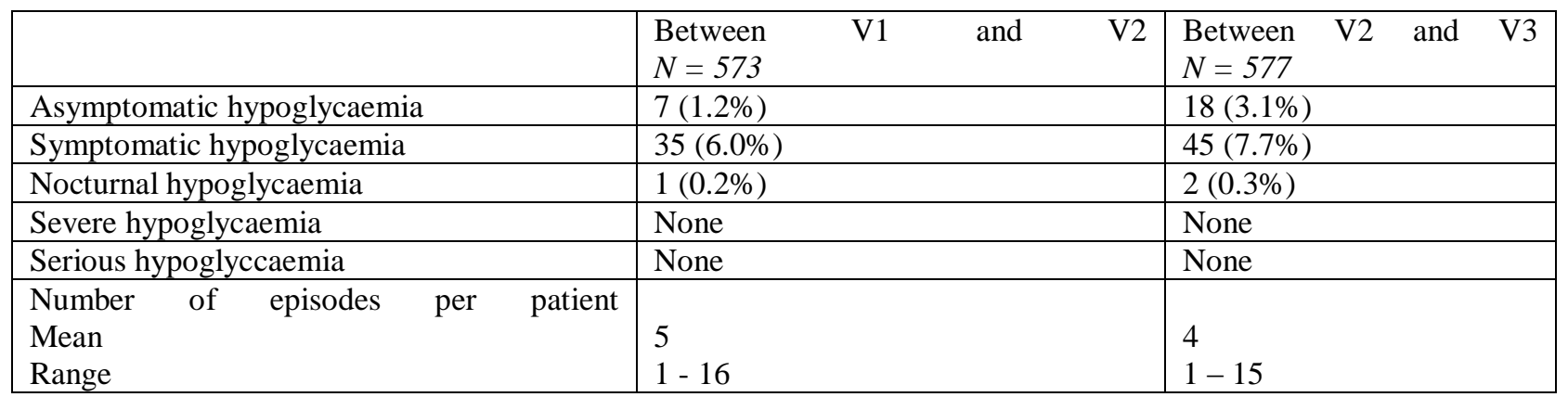

Table 6:-Hypoglycaemic episodes.

Data are presented as number of patients presenting at least one episode.

Differences in the occurrence of hypoglycaemic episodes between the 2 groups are described in table 7:-

\begin{tabular}{|l|l|l|l|l|}
\hline Group & $\begin{array}{l}\text { Between V1 and } \\
\text { V2 } \\
\text { (Symptomatic) }\end{array}$ & $\begin{array}{l}\text { Between V1 and } \\
\text { V2 } \\
\text { (Asymptomatic) }\end{array}$ & $\begin{array}{l}\text { Between V2 and } \\
\text { V3 } \\
\text { (Symptomatic) }\end{array}$ & $\begin{array}{l}\text { Between V2 and } \\
\text { V3 } \\
\text { (Asymptomatic) }\end{array}$ \\
\hline $\begin{array}{l}\text { Free combination of } \\
\text { Glimepiride and Metformin }\end{array}$ & 20 & 4 & 31 & 14 \\
\hline $\begin{array}{l}\text { Fixed dose combination } \\
\text { (Glimepiride + Metformin) }\end{array}$ & 15 & 3 & 14 & 4 \\
\hline
\end{tabular}

Table 7:- differences in the occurrence of hypoglycaemic episodes between the 2 groups

Safety:-

Six adverse events in six individual patients were reported during the course of the study. Hypoglycaemia was reported in five patients, including two cases of recurrent symptomatic hypoglycaemia. In all cases, hypoglycaemia was considered to be related to glimepiride by the investigators. This led to discontinuation of glimepiride in two patients, one of whom left the study and the other continued on metformin monotherapy. Two of these hypoglycaemic events were of moderate intensity while the remaining three were of mild intensity. Dyspepsia of mild intensity was observed in one patient, which was considered to be related to metformin. All of these adverse events had resolved prior to the end of the study. 


\section{Discussion:-}

The objective of this study was to determine the effectiveness and tolerability of bitherapy with glimepiride and metformin in patients failing to achieve glycaemic goals on monotherapy alone. We observed that the proportion of patients achieving glycaemic control on bitherapy after six months of treatment was $46 \%$, which falls within the range of previous studies (44\% to $66 \%$ in a recent systematic review) (Esposito et al., 2011). This suggests that the benefits of such bitherapy observed in clinical trials can be achieved in real-world treatment settings in the absence of the constraints and interventional procedures that may artificially raise response rates in randomized clinical trials in a number of other indications (Patsopolos, 2011). This is consistent with other observational trials in T2DM which have demonstrated effectiveness rates consistent with data from clinical trials (Matheiu et al., 2013).

With regard to safety, the number of adverse events reported in the trial was low, and essentially restricted to cases of symptomatic hypoglycaemia. Two patients experiencing repeated episodes of symptomatic hypoglycaemia discontinued glimepiride treatment. The incidence of hypoglycaemia reported in the study was relatively low, <5\% for asymptomatic hypoglycaemia and $7.7 \%$ for symptomatic hypoglycaemia. This may in part reflect incomplete reporting, notably for asymptomatic hypoglycaemia, due to a recall bias, since these events were documented retrospectively at the three-monthly study visits. Without documented and regular measures of blood glucose, asymptomatic hypoglycaemia is likely to go undocumented. Such under-reporting of hypoglycaemia has been reported previously in other pragmatic trials in T2DM (Matheiu et al., 2013). In clinical trials, where patients were instructed to use a glucose meter and patient diary to record hypoglycaemic events in real time, the rate of symptomatic hypoglycaemia in patients receiving metformin and glimepiride bitherapy was reported to be over $20 \%$ (Charpentier et al., 2001). Although hypoglycaemia is the principal adverse drug reaction associated with this class of OAD (Inzucchi et al., 2015), glimepiride is considered as carrying a lower risk of hypoglycaemia and weight gain for an equivalent benefit with respect to glycaemic control than earlier generation sulphonylureas (Ingle and Talele 2012, Basit et al., 2012). Indeed, in this real-world study, changes in weight following initiation of bitherapy were not significant.

Over the course of the study, the dosing of bitherapy was changed in around $40 \%$ of patients at Visit 2 and in around $10 \%$ at Visit 2. The majority of these changes represented increases in the dose of glimepiride or of metformin (or both). In part, this may reflect the recommended posology of glimepiride, which is to start on a low dose (1 - $2 \mathrm{mg}$ ) and then to titrate up as a function of glycaemia changes up to the maximum daily dose of 6 mg, while maintaining the metformin dose stable. Changes in metformin dose and frequency may indicate intensification of treatment at these study visits due to failure to reach HbA1c targets. On the other hand, the proportion of patients who were moved to OAD tritherapy (15.7\% of patients) or who were prescribed add-on insulin (1.6\%) was low, in spite of the fact that half of the patients in the study did not reach the desired HbA1c target after six months of treatment.

Around half of patients were receiving a fixed-dose combination of glimepiride and metformin. Such fixed-dose combinations of OADs for use in bitherapy offer better adherence to therapy and, as a consequence, help optimise glycaemic control (Bain, 2009). Dose modifications at Visits 2 and 3 in patients taking fixed-dose combinations were less frequent than in patients taking free combinations of glimepiride and metformin.

This study has a number of strengths and limitations. The strengths include the large number of patients included in the study and the low rate of exclusion of patients from the analysis due to protocol violations or loss to follow-up. In addition, the pragmatic study design should ensure that the findings reflect the impact of bitherapy with glimepiride and metformin in participating countries in the Middle East. With regard to limitations, potentially under-reporting of hypoglycaemia has already been discussed. In addition, the study design provided some scope for inclusion bias, in that participating physicians only switched patients to bitherapy once they had agreed to participate in the study. This may have resulted in patients being switched to glimepiride and metformin who would otherwise not have been switched (or switched to another bitherapy). The observation that certain patients included had been treated with metformin monotherapy for many years would suggest that some such inclusion bias may have occurred. In addition, the study was performed by GPs only and the absence of information on patients with diabetes who are managed by specialists, and who are potentially more severe or more difficult to control, may give an overoptimistic picture of the effectiveness of treatment in the overall population of patients with diabetes in participating countries.

In conclusion, this pragmatic real-world study demonstrated that the effectiveness and tolerability of bitherapy with glimepiride and metformin in patients with T2DM in the Gulf area is consistent with the findings of randomized 
clinical trials. Nonetheless, patients who do not achieve the HbA1c target of $\leq 7 \%$ by the end of the study are infrequently moved to a more intense treatment regimen, suggesting that further physician education on the relevance of treat-to-target therapeutic strategies in T2DM would be beneficial.

\section{Acknowledgements:-}

This study was supported by Sanofi. The authors thank all the study investigators for their contribution. The authors also acknowledge writing and editing assistance in developing this manuscript by FOXYMED, funded by Sanofi.

\section{Conflict of Interest:-}

Sameer Mohammed Al Shammari has a scientific collaboration with Sanofi, he however has no conflict of interest with the writing of this paper. Mohammed Ismail is employed by Sanofi.

\section{Funding:-}

This study was funded by Sanofi Gulf Countries 